\title{
A longitudinal observational study of the dynamics of Mycoplasma bovis antibodies in naturally exposed and diseased dairy cows
}

\author{
Mette B. Petersen, ${ }^{1}$ Jeanette Pedersen, ${ }^{2}$ Dinah L. Holm, ${ }^{3}$ Matthew Denwood, and Liza R. Nielsen \\ Department of Veterinary and Animal Sciences, Faculty of Health and Medical Sciences, University of Copenhagen, 1870 Frederiksberg C, \\ Denmark
}

\begin{abstract}
Mycoplasma bovis is an important pathogen causing disease and substantial economic losses in cattle. However, knowledge of the dynamics of antibody responses in individual cows in the face of an outbreak is currently extremely limited. The use of commercial antibody tests to support clinical decision-making and for surveillance purposes is therefore challenging. Our objective was to describe the dynamics of $M$. bovis antibody responses in 4 Danish dairy herds experiencing an acute outbreak of $M$. bovis-associated disease, and to compare the antibody dynamics between dairy cows with different disease manifestations. A total of 120 cows were examined using a standardized clinical protocol and categorized into 4 disease groups: "mastitis," "systemic," "nonspecific," and "none." Paired blood and milk samples were collected and tested using a commercial $M$. bovis antibody-detecting ELISA. Plots of raw data and generalized additive mixed models with cow and herd as random effects were used to describe serum and milk antibody dynamics relative to the estimated time of onset of clinical disease. Cows with mastitis had high optical density measurement (ODC\%) of antibodies in both milk and serum at disease onset. The estimated mean ODC\% in milk was below the manufacturer's cut-off for the other groups for the entire study period. The estimated mean serum ODC\% in the "systemic" group was high at onset of disease and stayed above the cut-off until $65 \mathrm{~d}$ after disease onset. However, the lower 95\% confidence interval (CI) for the mean ODC\% was only above the manufacturer's cut-off between 7 and $17 \mathrm{~d}$ after onset of disease. The CI of the "systemic" and "none" groups did not overlap at any time between the day of disease onset and $65 \mathrm{~d}$ after

Received December 22, 2017.

Accepted April 6, 2018.

${ }^{1}$ Corresponding author: mbp@sund.ku.dk

${ }^{2}$ Present affiliation: Øster Jølby Dyreklinik, Udvejen 3, 7950 Erslev, Denmark.

${ }^{3}$ Present affiliation: Dyrlæge Center Vest, Herningvej 74 A, 6950 Ringkøbing, Denmark.
\end{abstract}

disease onset, and the estimated mean ODC\% for both the "nonspecific" and "none" groups were generally below the cut-off for the majority of the study period. In conclusion, the serum antibody responses were highly dynamic and showed a high level of variation between individual cows. This strongly suggests that serology is unlikely to be useful for individual diagnosis of M. bovisassociated disease in dairy cows. However, it might still be useful for herd- or group-level diagnosis. Antibodies in milk were only increased in cows with $M$. bovis mastitis, indicating that milk antibody measurements only have diagnostic utility for cows with mastitis.

Key words: Mycoplasma bovis, ELISA, antibody, BioX Bio K 302, dairy cow

\section{INTRODUCTION}

Mycoplasma bovis is an infectious disease of cattle that is associated with a diverse spectrum of clinical signs and substantial production losses worldwide (Nicholas, 2011). In cows, $M$. bovis typically causes mastitis, arthritis, and pneumonia (Maunsell et al., 2011), whereas pneumonia, otitis media, and arthritis are more commonly seen in calves (Maunsell and Donovan, 2009). The preferred method of diagnosing $M$. bovis has historically been bacteriological culture of body fluids (e.g., milk and joint fluids) or swabs (e.g., eye and nasal swabs) from individual animals, as well as bacteriological culture of bulk tank milk for herdlevel diagnosis. Even though PCR is becoming more frequently used due to reductions in cost and processing time (Wawegama and Browning, 2017), these methods can have low sensitivity in practice due to intermittent shedding patterns, and it may be difficult to obtain the best sample materials from infected animals on which to apply these tests. Therefore, there is increasing interest in using serology for M. bovis diagnostics.

In Denmark, serological assays such as ELISA are frequently used for testing dairy cows, because they are inexpensive and convenient, especially if applicable to milk samples routinely collected for other purposes. Two commercial ELISA kits are available from BioX 
Diagnostics (Rochefort, Belgium), and one (BioX Bio K 260) has been shown to have little correlation with the occurrence of disease or with PCR and bacterial culture results (Szacawa et al., 2015, 2016). However, these studies aimed mainly to compare antibody measurements to other diagnostic tests using cross-sectional study designs that are not suitable for the assessment of dynamics and persistence of antibodies. Nielsen et al. (2015) found that for the BioX Bio K 302 ELISA, it might be beneficial to raise the cut-off for herd-level diagnosis to an optical density coefficient (ODC\%) of 50 in bulk tank milk to increase the specificity, but no field study evaluations of cut-off values at the animal level have been published. Therefore, a cut-off of 37 ODC\%, as recommended by the manufacturer, is used in practice despite the lack of substantial documentation for the validity of this threshold.

Appropriate interpretation of ELISA test results requires knowledge of the dynamics and duration of excretion of antibodies against $M$. bovis relative to the time of infection and onset of associated disease. However, as recently pointed out by Hazelton et al. (2018), there is currently a limited understanding of the dynamics of antibodies directed against $M$. bovis in terms of time to seroconversion and longevity in naturally exposed cattle. The antibody response has been shown to remain high in both milk and serum for up to 20 wk after either experimental inoculation in the udder or naturally occurring $M$. bovis-associated mastitis (Boothby et al., 1987; Byrne et al., 2000). However, the $M$. bovis antibody response to systemic disease such as arthritis has not yet been fully described. Work in calves vaccinated against $M$. bovis at 3 wk of age with an experimental vaccine showed that the animals appeared to seroconvert within $14 \mathrm{~d}$ and that a high IgG level was maintained for at least $42 \mathrm{~d}$ in serum (Nicholas et al., 2002). Apart from this work in individual animals, serology has been suggested to be useful for herd-level diagnostics (Martin et al., 1990; Le Grand et al., 2002).

Further investigation of ELISA test result patterns in milk and serum from naturally exposed and diseased dairy cows is therefore warranted, in particular to understand how the ELISA response can be expected to develop over time in animals with varying clinical signs of $M$. bovis-associated disease compared with exposed animals without overt clinical signs. The objective of this study was therefore to describe the temporal dynamics of antibody responses to $M$. bovis in serum and milk taken from individual dairy cows in herds experiencing an $M$. bovis disease outbreak, with a particular emphasis on differences in these patterns between groups of animals exhibiting different disease manifestations.

\section{MATERIALS AND METHODS}

\section{Study Design}

This study was a longitudinal observational study in Danish dairy cattle herds. To fulfill the objective of describing dynamics of antibody responses in exposed animals, only herds experiencing acute outbreaks of $M$. bovis-associated disease within the study period were eligible for inclusion. This was based on the likely presence of $M$. bovis-associated disease as diagnosed by the herd advisory veterinarian based on positive PCR or ELISA test results, and an outbreak was defined as having several animals with clinical signs of mastitis (Maunsell et al., 2011), arthritis, subcutaneous swelling of the limbs (Henderson and Ball, 1999; Wilson et al., 2007), pneumonia (Maunsell et al., 2011), or combinations thereof, and positive $M$. bovis ELISA or PCR tests.

Four herds were identified as matching these criteria and permission was obtained from each herd to undertake an outbreak investigation. Each herd was visited 5 times, at approximately 3-wk intervals, during the period from July 1, 2015, to April 5, 2016. The herd visits were initiated as closely as possible following the presumed date of onset of the disease outbreak. At all visits, the aim was to assess the clinical status using a standard protocol and to collect paired blood and milk samples from selected individual cows. Where possible, the same animals were sampled at each visit. As many repeated samples as possible were obtained from as many individual animals as the project budget would allow. When it was not possible to resample the same animals, new animals were sampled.

\section{Study Population}

All 4 of the identified herds had a history of sudden onset of M. bovis-related clinical signs in cows or calves, and several strongly positive ELISA or PCR test results for $M$. bovis (Table 1). During the study period, one or more cows from all study herds tested positive at least once by ELISA or PCR. Detailed farm information obtained before and after enrollment is given in Table 1 .

Although bacterial cultures were not included in the study design due to financial constraints, a few calves were euthanized due to severe disease and autopsies were performed. A calf from herd 2 had chronic degenerative arthrosis in several joints and bronchopneumonia with overlying pleuritis. Mycoplasma bovis was cultured from joint fluid, and joint fluid and lung tissue were found positive for $M$. bovis by PCR. Two calves autopsied from herd 4 had chronic omphalitis, bronchopneumonia, synovitis in several joints, and bilateral 
otitis media. Mycoplasma spp. were cultured from both calves and typed by PCR to be $M$. bovis. From herd 3, 2 calves were autopsied and arthritis and otitis media were found in both animals, but no bacterial pathogens were isolated.

\section{Sample Collection}

The project budget allowed for the inclusion of approximately 80 cows in total, with each being tested 5 times. However, during an outbreak of M. bovis, it is likely that some cows would be culled due to clinical disease; therefore, we allowed for more cows to be included at the first or consecutive visits. Therefore, a minimum of 20 cows were identified from each farm during the first herd visit. The cows were sampled strategically to ensure that a sufficient number of cows with clinical suspicion of $M$. bovis-associated disease were included along with a sample of cows without such clinical disease suspicion. New cows suspected of having $M$. bovis-associated disease at subsequent visits were also included. The rationale behind this nonrandom sampling strategy was to maximize the chances of including sufficient numbers of animals with and without different clinical signs.

At each visit, the selected cows were subject to a clinical examination focusing on the respiratory system, udder, and musculoskeletal system using a standardized clinical protocol (Appendix Table A1). The clinical examination was done by 1 of 3 veterinarians,
2 of whom had collaboratively developed the clinical protocol. At least one of these was present at each visit, and the use of the clinical protocol was demonstrated for the benefit of the third veterinarian who had not helped to develop the protocol.

In addition to the clinical examination, a venous blood sample was collected in a 10-mL plain Vacutainer tube (Kruuse, Denmark), and one composite milk sample was collected in a bronopol-coated tube from each cow. Samples were stored in a cool environment, and delivered to Eurofins Steins Laboratory (Vejen, Denmark) within $36 \mathrm{~h}$.

\section{Laboratory Test Methods}

All samples were analyzed for antibodies directed against $M$. bovis using the commercial kit BioX Bio K 302 (BioX Diagnostics). The assay was performed according to the manufacturer's instructions for use. The optical density coefficient was calculated as follows:

$$
\begin{gathered}
\mathrm{ODC} \%=\left(\mathrm{OD}_{\text {sample }}-\mathrm{OD}_{\text {negative control }}\right) / \\
\left(\mathrm{OD}_{\text {positive control }}-\mathrm{OD}_{\text {negative control }}\right) \times 100 \%,
\end{gathered}
$$

where OD is the optical density measured by the ELISA reader of each test sample and the positive and negative control on the sample ELISA plate. An ODC $\% \geq 37$ was considered positive, as recommended by the manufacturer. The manufacturer has reported sensitivity and specificity of the test to be $100 \%$ based

Table 1. Summaries of the 4 Danish dairy herds in the study and results of different diagnostic tests performed before and during the study period from July 1, 2015, to April 5, 2016

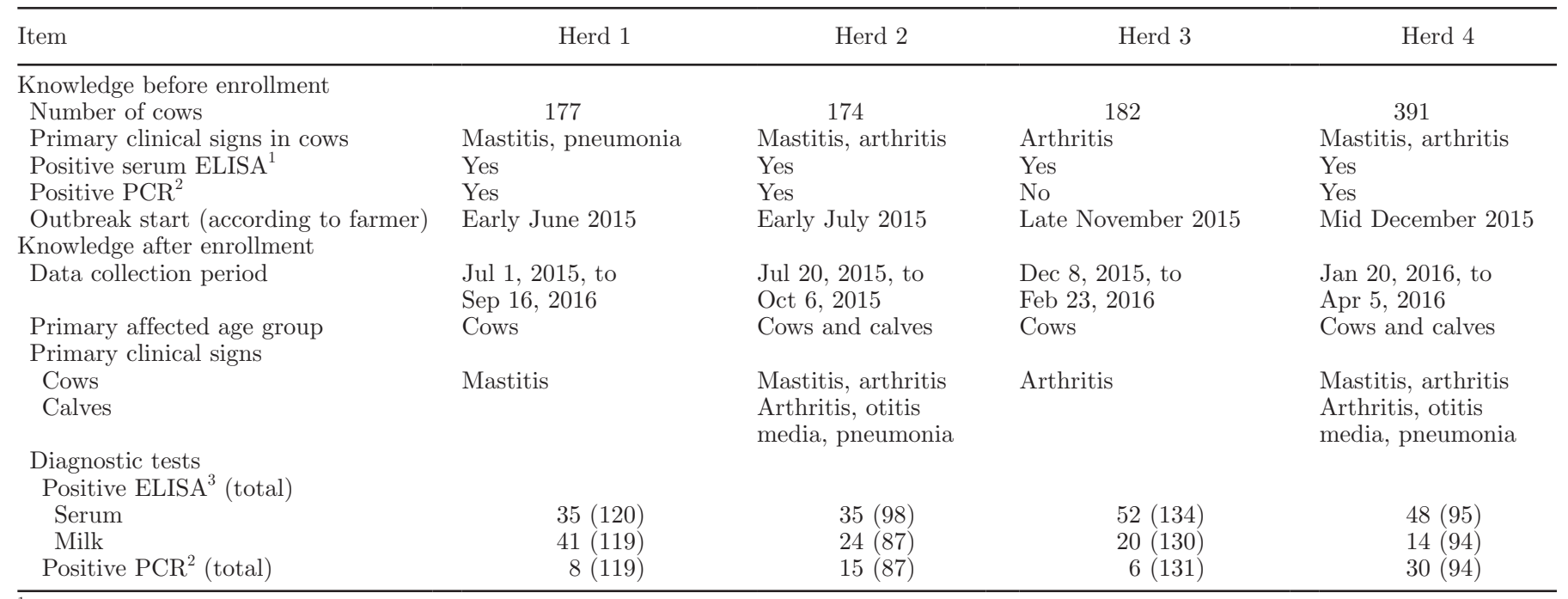

${ }^{1}$ Optical density coefficient (ODC\%) values $\geq 37$ for Mycoplasma bovis antibodies in BioX Bio K 302 or 260 (BioX Diagnostics, Rochefort, Belgium).

${ }^{2}$ Composite milk samples; cycle threshold (Ct) value $\leq 37$ for $M$. bovis in PathoProof Mastitis Major-3 (Thermo Scientific, Waltham, MA). ${ }^{3} \mathrm{ODC} \%$ values $\geq 37$ for Mycoplasma bovis antibodies in BioX Bio K 302 (BioX Diagnostics). 
on a small sample of experimentally infected calves and negative control calves (BioX Diagnostics, 2017). However, lower accuracy has been reported under field conditions (Wawegama et al., 2016; Hazelton et al., 2018).

Milk samples were analyzed for presence of $M$. bovis DNA with the commercial PCR kit PathoProof Mastitis Major-3 assay (Thermo Scientific, Waltham, MA) at Eurofins Steins Laboratory. A cycle threshold (Ct) value $\leq 37$ was considered positive in the PCR, as recommended by the manufacturer.

\section{Grouping of Cows for Analysis}

Following the completion of the data collection period, individual animals were categorized into disease groups to facilitate stratification of descriptive statistics and modeling of antibody response dynamics. The 3 primary groups were as follows: (1) likely $M$. bovis-associated mastitis (MAST); (2) likely M. bovisassociated systemic disease (SYS); and (3) no disease believed to be associated with $M$. bovis (ND). In addition, a fourth group was defined for the remaining animals that could not be reliably assigned to groups 1 to 3: (4) disease that is not typically associated with $M$. bovis, but where $M$. bovis could not reasonably be excluded (nonspecific, NS).

Classification was based on the recorded clinical signs and photographs taken of the selected cows during the herd visits, according to the specific inclusion criteria as follows:

(1) MAST: cows with recordings of mastitis, defined as any visual abnormality in the milk and a $M$. bovispositive milk PCR at one or more herd visits.

(2) SYS: cows with clinical signs indicating systemic spread of M. bovis. All cows in this group had arthritis, subcutaneous edema in the limbs, or both, at one or more herd visits. In addition to these clinical signs from the musculoskeletal system, 3 cows had clinical signs of respiratory disease, 2 had drooping ears, and 12 had mastitis.

(3) ND: cows with no clinical signs, and cows with only clinical signs that are not likely to be associated with $M$. bovis. These included hock lesions, wounds, claw lesions, cows with a dry quarter, and lameness without joint swelling. However, due to the presence of clinical signs in co-managed animals, it is assumed that these animals were exposed to the pathogen.

(4) NS: cows that had clinical signs that are not typical for $M$. bovis, but where $M$. bovis could not be excluded. Clinical signs in this group were minor, short-term limb swellings, or mastitis (without an $M$. bovis PCR positive test), mild or short-term respiratory signs, keratoconjunctivitis, and abscesses.
All 4 groups were modeled separately to facilitate the focus on the differences of the temporal dynamics between groups. Twelve cows fulfilled the criteria for being in both the MAST and SYS groups, hereafter referred to as "dual-syndrome" cows. They are included in both the MAST and SYS groups for analysis of antibody dynamics.

\section{Primary Explanatory Variable of "Days from Disease Onset"}

The primary explanatory variable for the longitudinal analysis was the number of days between the estimated disease onset in the individual cow and the sample date for the relevant ELISA result. It was not possible to determine this exactly but was estimated from the first date of observed clinical signs in each cow. To make this as accurate as possible, records from the farmers' electronic disease recording system and written records from the consulting veterinarians were used where available. In $39 \%$ of the cows, a similar diagnosis as the one given at the herd visit was recorded by farmer or veterinarian within 1 wk before the herd visit, and this day was then set as the day of disease onset. In $61 \%$ of the cows, the day of disease onset was recorded as the date of the herd visit at which the clinical sign was detected for the first time. To be able to make comparisons between all groups, cows in the ND group were assigned a representative imputed date that was sampled from the distribution of estimated disease onset dates from the cows in the MAST and SYS groups within the same herd.

\section{Modeling of Antibody Responses}

To investigate the potentially nonlinear temporal patterns of antibody responses, we selected an explorative approach to avoid imposing a prespecified functional form to the antibody dynamics over time. Four separate generalized additive mixed models (GAMM) were used for the 2 outcomes (serum and milk) in each of the 4 disease groups; that is, 8 models in total. The ODC\% was log-transformed to improve the normality of the residuals. To allow log-transformation of a small number of ELISA values that were recorded as numerically 0 , a fixed constant of 1 was added to all ODC\%. The data used for the models were restricted to the most relevant periods from $21 \mathrm{~d}$ before disease onset to $80 \mathrm{~d}$ after disease onset (Figure 1). As well as reflecting the most relevant period in relation to the presumed date of onset of clinical signs, this procedure also ensured that time periods were limited to those with observations from a minimum of 3 cows. 

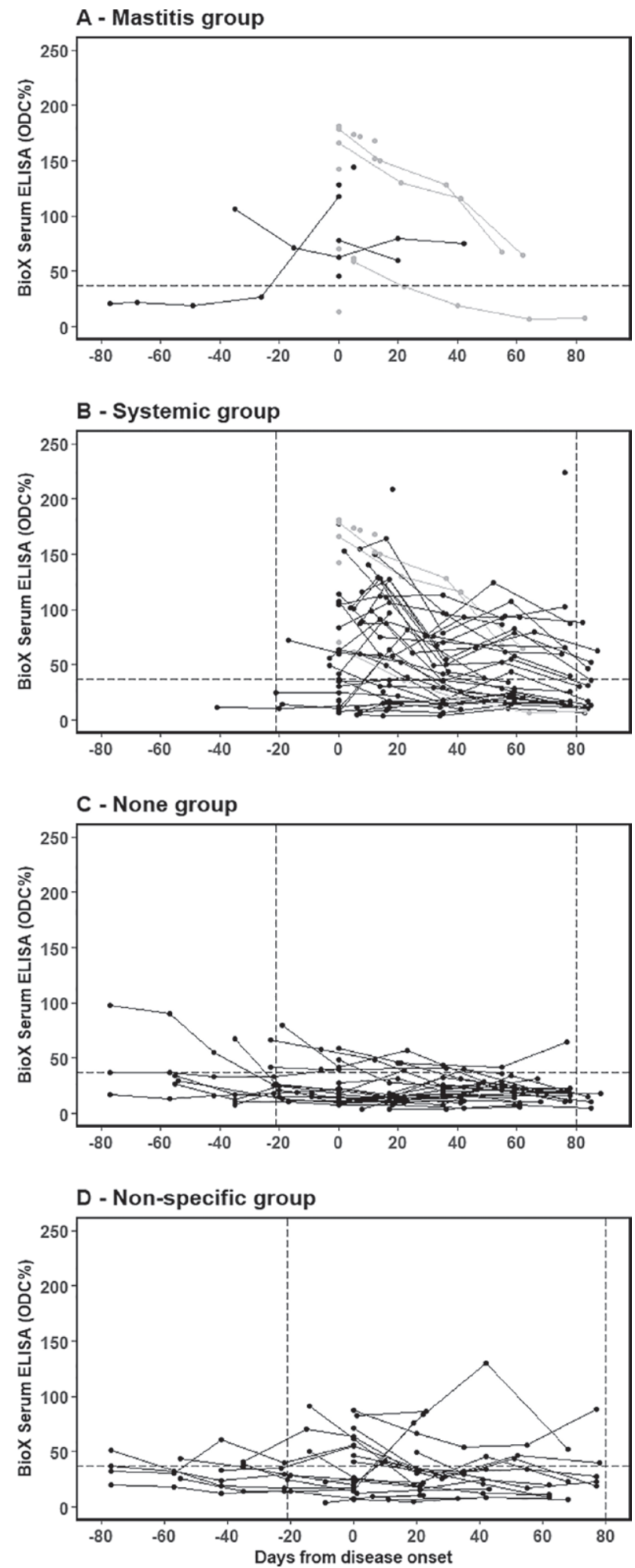

Figure 1. Distribution of serum ELISA (BioX ELISA Bio K302; BioX Diagnostics, Rochefort, Belgium) measurements for antibodies directed against Mycoplasma bovis in the 4 disease groups of dairy cows from 4 Danish herds: Mastitis = likely Mycoplasma bovis-associated mastitis; Systemic $=$ likely $M$. bovis-associated systemic disease; None $=$ no disease believed to be associated with $M$. bovis; Nonspecific $=$ disease that is not typically associated with $M$. bovis, but where $M$. bovis could not reasonably be excluded. Dual-syndrome cows (fulfilled criteria for being in the mastitis and systemic groups) are colored in gray. Horizontal dashed lines show the recommended ELISA cut-off (37 ODC\%). Vertical dashed lines indicate the temporal limits for inclusion in the modeling of antibody response dynamics ( $\mathrm{d}-21$ and 80 relative to disease onset). ODC $\%=$ sample optical density coefficient. Results from the same cow are linked by lines. 
Table 2. Number of cows and samples in each disease group, ${ }^{1}$ stratified by herd

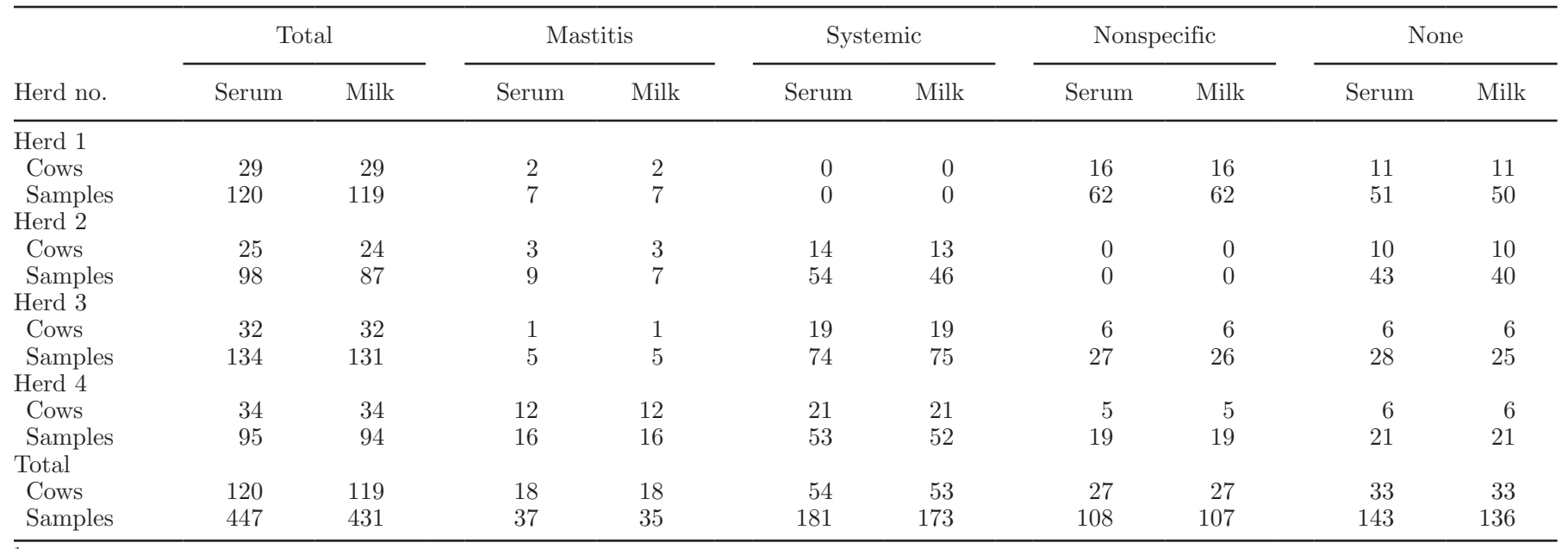

${ }^{1}$ Mastitis = likely Mycoplasma bovis-associated mastitis; Systemic = likely M. bovis-associated systemic disease; Nonspecific = disease that is not typically associated with $M$. bovis, but where $M$. bovis could not reasonably be excluded; and None = no disease believed to be associated with $M$. bovis.

A thin plate regression spline was used to fit the nonlinear effect of day from disease onset, and random effects of cow identification number (cow) and herd identification number (herd) were included to account for repeated samples from the same cow and clustering of cows within herds. Confidence intervals for the predicted mean ODC\% values (with random effects of herd and cow set to zero) were calculated and plotted for each disease group and outcome. Model fit was assessed for each model by evaluating the distribution of the residuals. All data management and analyses were done in $\mathrm{R}$ version 3.2.2 ( $\mathrm{R}$ Core Team, 2016), using the "gamm" function in the "mgcv" package to implement the GAMM (Wood, 2011).

\section{RESULTS}

\section{Descriptive Statistics}

In total, 120 cows were enrolled in the study, and 447 blood samples and 431 milk samples were collected. See Table 2 for detailed information about number of cows and samples stratified by herd.
Cows were sampled an average of 3.7 times, with herd 4 having the lowest average number of observations per cow (2.8; Table 3). This reflects the fact that many cows with mastitis were slaughtered or culled soon after disease onset in herd 4 . It was not possible to collect milk samples from cows that were dried off during the study period, but clinical examinations and blood collections continued during the dry period. Reasons for loss from the study were culling, slaughter, or movement to another property for drying-off.

All but one of the cows in the MAST group had a serum result that was well above the manufacturer's cut-off on the day of disease onset, and most of the cows declined rapidly in ODC\% following the date of onset (Figure 1A).

A total of 27 out of 54 (50\%) cows with systemic $M$. bovis-associated disease (SYS group) had serum results that were above the cut-off for the entire study period (Figure 1B). A total of 15 out of $54(28 \%)$ cows in the SYS group were not above the cut-off at any point in time, and 12 of the $54(22 \%)$ cows had some samples above and some below the cut-off during the study period. The antibody responses declined slowly for most

Table 3. Average number of samples per cow and the distribution of samples per cow, stratified by herd

\begin{tabular}{lcrrrrr}
\hline & & \multicolumn{5}{c}{ No. of samples } \\
\cline { 3 - 7 } Herd & Average & 1 & 2 & 3 & 4 & 5 \\
\hline 1 & 4.1 & 0 & 6 & 1 & 5 & 17 \\
2 & 4 & 4 & 1 & 2 & 4 & 14 \\
3 & 4.2 & 4 & 0 & 1 & 8 & 19 \\
4 & 2.8 & 13 & 3 & 3 & 8 & 7 \\
Total & 3.7 & 21 & 10 & 7 & 25 & 57 \\
\hline
\end{tabular}


of the cows, ending up below the cut-off before or at the last visit.

A total of 22 out of $33(67 \%)$ cows in the ND group had serum results that were below the cut-off for the entire study period (Figure 1C). One out of $33(3 \%)$ cows was above the cut-off for the entire study period, and 10 out of $33(30 \%)$ cows had some samples above and some below the cut-off during the study.

A total of 2 of $27(7 \%)$ cows in the NS group had serum results that were above the cut-off for the entire study period (Figure 1D). A total of 8 out of $27(30 \%)$ cows were below the cut-off at all points in time, and 17 of the $27(63 \%)$ cows had samples above and below the cut-off during the study period. On average, the ODC\% values in the NS group were lower than in the SYS group.

The overall pattern was less consistent for milk samples than for serum samples in the MAST group. A total of 8 of 18 (44\%) cows with M. bovis mastitis had milk results that were above the cut-off for the entire study period. A total of 7 of 18 (39\%) cows had milk results that were below the cut-off for the entire study period, and 2 of 18 (11\%) cows had milk results above and below the cut-off during the study period (Figure 2A).

A total of 11 of $53(20 \%)$ cows in the SYS group had milk results that were above the cut-off for the entire study period (Figure 2B) and 5 of these cows also had M. bovis mastitis. A total of 22 of 53 (42\%) cows had milk results that were not above the cut-off at any time, and 20 of the $53(38 \%)$ cows had milk results above and below the cut-off during the study period.

A total of 23 cows of $33(70 \%)$ cows in the ND group had milk results that were below the cut-off for the entire study period (Figure 2C). A total of 3 of 33 (9\%) cows had milk results above the cut-off for the entire study period and 7 of the $33(21 \%)$ cows had milk results above and below the cut-off during the study period.

A total of 4 of $27(15 \%)$ cows in the NS group had milk results that were above the cut-off for the entire study period (Figure 2D), whereas 14 of $27(52 \%)$ cows had milk results that were below the cut-off at all times, and 9 of the $27(33 \%)$ cows had milk results both above and below the cut-off during the study period.

\section{Modeling Results}

Eight separate models were used for the 2 outcomes (log-transformed milk and serum ODC\% values) and 4 disease groups, although meaningful results could not be obtained for the MAST group models of either milk or serum samples due to sparse and highly variable data (Figures 1A and 2A). Visual inspection of residual plots indicated an acceptable model fit for the remaining 6 models, results of which are presented below.

The estimated number of degrees of freedom for the smooth term describing the effect of day from disease onset indicates the complexity of the function, with values of 1 indicating a simple linear function. Based on the estimated degrees of freedom, substantial nonlinearity was evident in the SYS group for both milk and serum and in the ND group for milk. The standard deviation associated with the random effect of cow was larger than the residual standard deviation in all the models, and the random effect attributed to herd was generally smaller than that attributed to cow. However, the random effect of herd is likely to be underestimated because of the small number of herds included in the study, so some of the true differences between herds are likely included in the cow random effect estimate. We therefore considered only the combined effect of cow and herd relative to the residual standard deviation as a meaningful comparison (Table 4).

The estimated mean ODC\% in serum in the SYS group was estimated to be high at the time of disease onset and remained above the manufacturers' cut-off until $65 \mathrm{~d}$ after disease onset (Figure 3, horizontal dashed line). The 95\% CI followed the same pattern but indicated that the mean ODC\% was clearly above the cut-off only between 7 and $17 \mathrm{~d}$ after disease onset in this group. The estimated mean ODC\% with $95 \%$ CI in the ND group were below the cut-off for most of the period, and there was no overlap of CI between the SYS and ND groups between 1 wk before day of disease onset and approximately $68 \mathrm{~d}$ after disease onset (Figure 3). The CI of the NS group overlapped with that of the ND group during the whole study period and only differed markedly from the SYS group according to the CI between 7 and $20 \mathrm{~d}$ after disease onset.

Estimated ODC\% in milk was below the cut-off for all disease groups for the entire period, with wide 95\% CI that overlapped substantially between disease groups throughout the study period (Figure 4).

\section{DISCUSSION}

This observational study is the first to illustrate and analyze the dynamics of the antibody response directed against $M$. bovis from milk and serum in naturally exposed and diseased dairy cows. The key findings were that the antibody responses varied markedly between cows and were very dynamic within individual cows. The serum antibody level generally declined within 2 mo following the onset of clinical disease, even in cows with clear clinical signs of systemic $M$. bovis disease. The antibody level in milk was only high if cows had mastitis. 

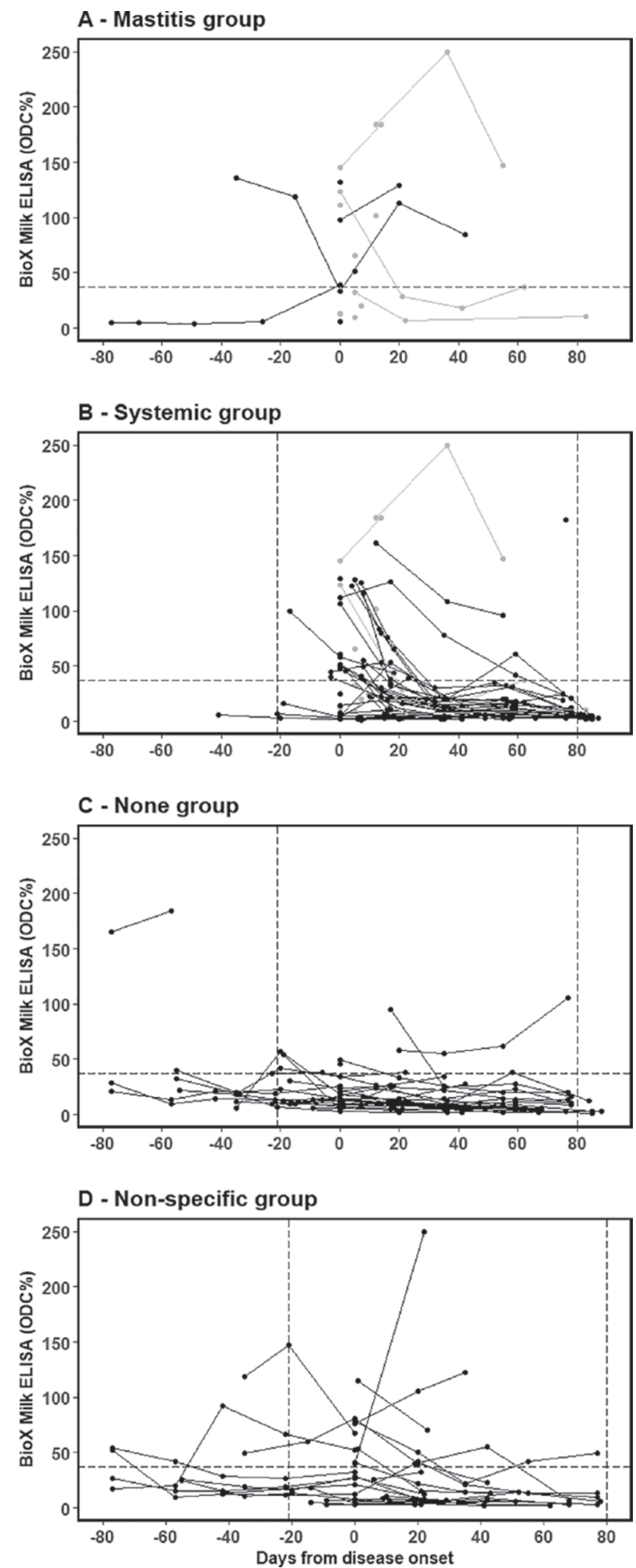

Figure 2. Distribution of milk ELISA (BioX ELISA Bio K302; BioX Diagnostics, Rochefort, Belgium) measurements for antibodies directed against Mycoplasma bovis in the 4 disease groups of dairy cows from 4 Danish herds: Mastitis = likely Mycoplasma bovis-associated mastitis; Systemic = likely $M$. bovis-associated systemic disease; None = no disease believed to be associated with $M$. bovis; Nonspecific $=$ disease that is not typically associated with $M$. bovis, but where $M$. bovis could not reasonably be excluded. Dual-syndrome cows (fulfilled criteria for being in the mastitis and systemic groups) are colored in gray. Horizontal dashed lines show the recommended ELISA cut-off (37 ODC\%). Vertical dashed lines indicate the temporal limits for inclusion in the modeling of antibody response dynamics $(\mathrm{d}-21$ and 80 relative to disease onset $)$. ODC $\%=$ sample optical density coefficient. Results from the same cow are linked by lines. Three observations with ODC\% >250 are shown as 250 ODC $\%$. 


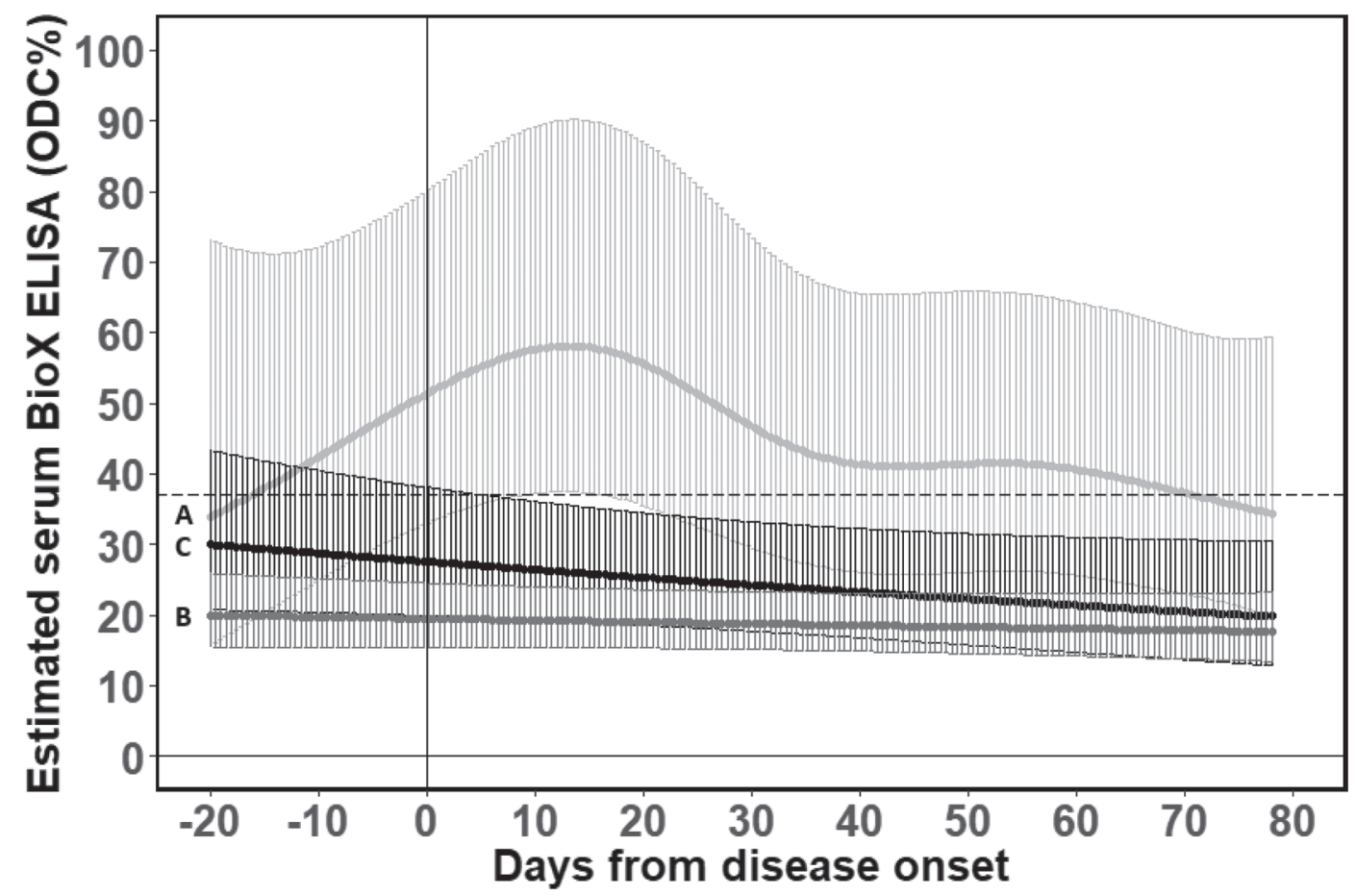

Figure 3. Estimated mean antibody response in serum (solid line) and 95\% CI (shaded area) as measured by the BioX ELISA Bio K302 (BioX Diagnostics, Rochefort, Belgium). A (light gray) = Systemic (likely Mycoplasma bovis-associated systemic disease); B (dark gray) = None (no disease believed to be associated with $M$. bovis); C (black) = Nonspecific (disease that is not typically associated with $M$. bovis, but where M. bovis could not reasonably be excluded). The dashed black line shows the recommended ELISA cut-off $(37$ ODC\%), where ODC\% $=$ sample optical density coefficient.

In calves, the serum IgG level has been found to increase between 1 and 2 wk after vaccination (Nicholas et al., 2002) and intratracheal inoculation of $M$. bovis bacteria (Howard et al., 1986). The antibody responses observed in this study from cows that responded to infection were high from the estimated day of disease onset, which indicates either that the cows had been infected for a week or two before becoming sick, or that antibody responses are faster and stronger in adult cows are than in young calves. In experimentally inoculated cows, antibody responses in serum and milk have been found to increase within 7 to $10 \mathrm{~d}$ from inoculation (Boothby et al., 1986; Byrne et al., 2005), indicating that the antibody responses might be slightly faster in cows than in calves. This also fits with the increasing pattern in estimated mean ODC\% that can be observed in Figure 3 between 0 and $14 \mathrm{~d}$ after disease onset.

\section{Antibodies Against M. bovis in Serum}

This study suggests that the level of serum antibodies generally increases in cows with $M$. bovis-associated

Table 4. Results of the final models with antibody optical density measurement (ODC\%) for Mycoplasma bovis in serum and milk as outcome in different disease groups ${ }^{1}$

\begin{tabular}{|c|c|c|c|c|c|c|}
\hline \multirow[b]{2}{*}{ Item } & \multicolumn{2}{|c|}{ Systemic } & \multicolumn{2}{|c|}{ None } & \multicolumn{2}{|c|}{ Nonspecific } \\
\hline & Serum & Milk & Serum & Milk & Serum & Milk \\
\hline \multicolumn{7}{|l|}{ Random effect (SD) } \\
\hline Combined cow/herd level & 0.88 & 1.14 & 0.49 & 0.80 & 0.62 & 1.08 \\
\hline Residuals & 0.43 & 0.65 & 0.35 & 0.47 & 0.46 & 0.53 \\
\hline \multicolumn{7}{|l|}{ Smooth term $\left(\right.$ edf $\left.^{2}\right)$} \\
\hline Days from onset of disease & 4 & 2.3 & 1 & 1.6 & 1 & 1 \\
\hline
\end{tabular}

${ }^{1}$ Systemic $=$ likely $M$. bovis-associated systemic disease; None $=$ no disease believed to be associated with $M$. bovis; Nonspecific $=$ disease that is not typically associated with $M$. bovis, but where $M$. bovis could not reasonably be excluded.

${ }^{2}$ Estimated degrees of freedom. 


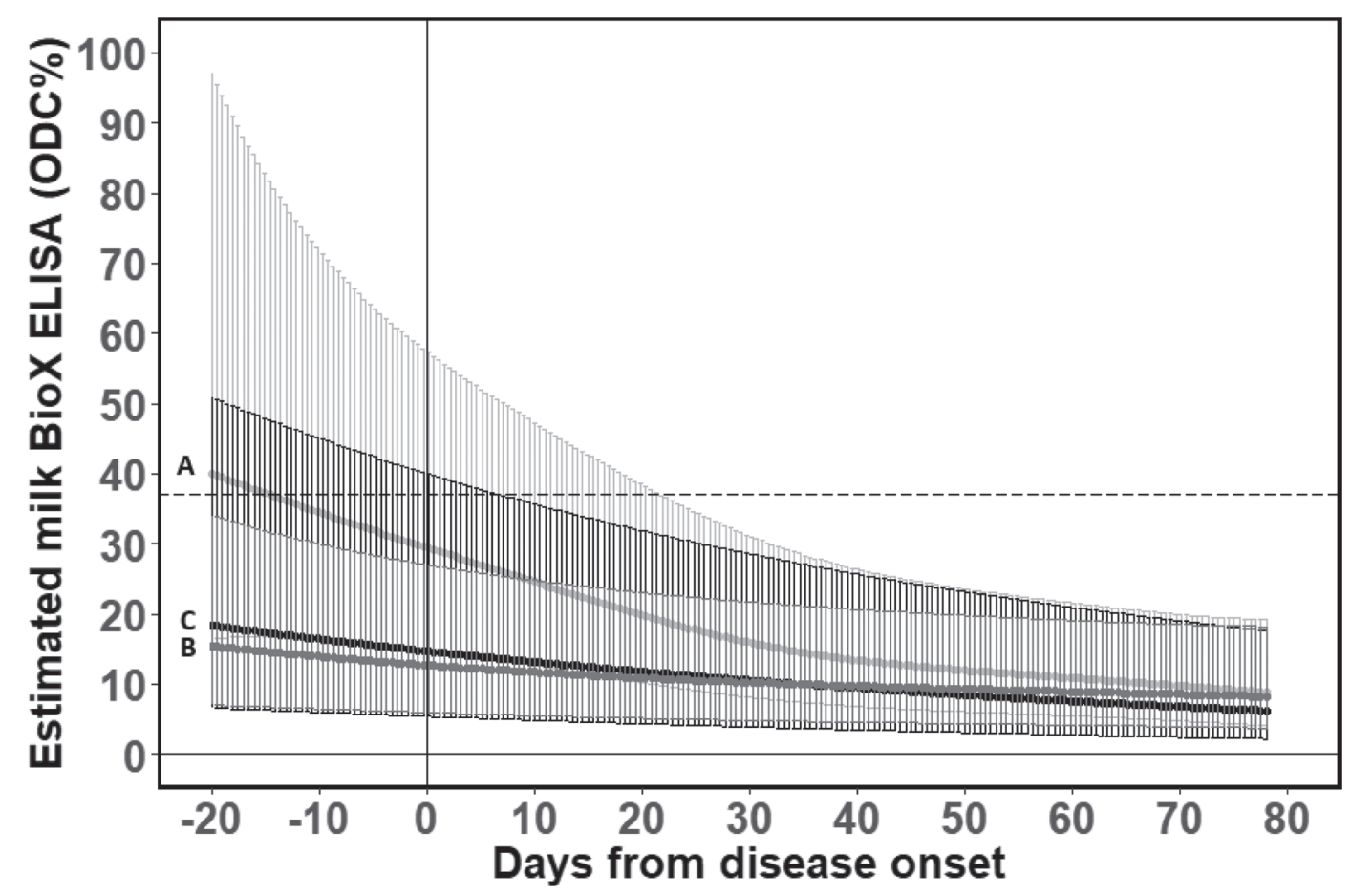

Figure 4. Estimated mean antibody response in milk (solid line) and 95\% CI (shaded area) measured by the BioX ELISA Bio K302 (BioX Diagnostics, Rochefort, Belgium). A (light gray) = Systemic (likely Mycoplasma bovis-associated systemic disease); B (dark gray) = None (no disease believed to be associated with $M$. bovis); C (black) = Nonspecific (disease that is not typically associated with $M$. bovis, but where $M$. bovis could not reasonably be excluded). The dashed black line shows the recommended ELISA cut-off $(37$ ODC\%), where ODC\% $=$ sample optical density coefficient.

disease around the time of disease onset (Figures 1A, $1 \mathrm{~B}$, and 3 ), which to our knowledge has not been described previously in naturally infected cows. For experimentally induced mastitis, the same tendency has been found (Boothby et al., 1987) but no such studies about arthritis are published. It appears that the ODC\% in the MAST group declined more quickly than the SYS group, but it is difficult to compare with so few samples in the MAST group. Although we were not able to follow all cows until their antibody response declined below the cut-off, a faster decline in antibodies was seen for $M$. bovis relative to other diseases for which antibody measurements are commonly used in cattle, such as Salmonella Dublin (Nielsen, 2003) and bovine virus diarrhea virus (Fredriksen et al., 1999).

The serum model for the SYS group was repeated excluding the 12 dual-syndrome cows (those with both mastitis and systemic disease) to assess the sensitivity of our results to the data from these animals. The shape of the curve was qualitatively similar, and the time period during which the confidence intervals did not overlap was the same, although the highest estimated mean declined by approximately 5 ODC $\%$ when excluding the 12 dual-syndrome cows. Unfortunately, it was not possible to repeat this procedure for the MAST group, because excluding the dual-syndrome cows left us with a sample size of only 6 animals in this group. However, we acknowledge that further investigation into specific features of antibody responses in dual-syndrome cows exhibiting both sets of clinical signs and how this differs from cows exhibiting only signs of mastitis would be relevant for future studies.

Some of the cows in the SYS group did not exhibit an antibody response (Figure 1B). This included 1 of the 12 dual-syndrome cows in this group, but that animal was tested only once. One explanation could be that the clinical signs in the nonresponding cows were not due to $M$. bovis infection but to some other pathogen or disease. We did not have the opportunity to diagnostically rule out other pathogens in this study. Another explanation for the lack of antibody responses could be that the ability to produce antibodies against $M$. bovis infection varies between individual cattle. It is unknown whether this difference between host responses is due to host (e.g., genetics) or pathogen factors, or a combination of the two. The antibody response to $M$. bovis has not been found to be correlated with treatment of bovine respiratory disease in calves (Rosendal and Martin, 1986) but no such studies have been conducted in cows. The antigenic variation in $M$. bovis 
bacteria is large and alterations of membrane surface lipoproteins occur during an outbreak, even between different subclones of the same strain (Bürki et al., 2015). This might result in different antibody responses in cows with similar clinical signs. Despite the lack of a clear explanation for the fact that some cows did not produce measurable antibodies, this finding is in agreement with others who found little correlation between antibody measurements and clinical signs in cows (Szacawa et al., 2016). However, in that study, the cows were not followed over time so it was not possible to explain the missing correlation.

Other authors have warned against using serology for individual M. bovis diagnosis (Maunsell et al., 2011). Martin et al. (1990) suggested that antibodies directed against $M$. bovis are not correlated with disease in individual calves but could be useful at the group level. Our results also indicate that the ODC\% are not well correlated with clinical disease in individual cows within herds with circulating M. bovis (Figure 1B). However, the estimated mean ODC\% with corresponding 95\% CI suggests that it is possible to use the average serum ODC\% of a group of diseased animals to assess whether the clinical signs in the herd are likely to be associated with $M$. bovis infection for a period of up to $65 \mathrm{~d}$ after disease onset in the affected animals (Figure 3 ). It is beyond the scope of this study to identify optimal testing scenarios for group or herd diagnostics, but the results of the raw data for mastitis (despite the fact that a model could not be fit to these data) indicate that such a screening tool would also be relevant for outbreaks with mastitis cases, and not only systemic disease.

\section{Antibodies Against M. bovis in Milk}

The level of antibodies against $M$. bovis in milk from cows other than those with $M$. bovis mastitis was low. Even if a cow had clear clinical signs of systemic spread of $M$. bovis; for example, arthritis with antibodies detectable in serum, almost no antibodies were detectable in the milk. In contrast, the cows with mastitis had high levels of antibodies in both milk and serum, and the cows in the SYS group with high milk antibodies were primarily cows with clinical signs of both systemic disease and mastitis. Byrne et al. (2000) found that antibodies in milk remained high for a longer period in quarters infected with $M$. bovis compared with quarters not infected in the same cow, suggesting that the antibody response to $M$. bovis in the udder is primarily a local immune response and, to a lesser extent, filtering of antibodies from serum to milk. Antibodies in milk can therefore only be used for diagnosis of cows with mastitis, not for cows with systemic disease without mastitis. In mastitis cases in other studies, antibodies in milk lasted for at least $40 \mathrm{~d}$ (Boothby et al., 1987; Byrne et al., 2000). We were not able to confirm this, as it was not possible to determine the duration of high antibody levels in milk in cows with mastitis due to culling or euthanasia of many of the cows with mastitis.

Our results explain why others have found limitations in the use of antibody measurements in bulk tank milk for diagnosis $M$. bovis at the herd level (Nielsen et al., 2015; Petersen et al., 2016; Parker et al., 2017). Usually cows with clinical signs of mastitis will not be milked into the bulk tank for delivery to the dairy and therefore do not contribute to the bulk tank milk antibody response. However, because the antibody level in milk after mastitis seems to last for at least $20 \mathrm{wk}$ (Boothby et al., 1987; Byrne et al., 2000), they might contribute after clinical signs or treatment have ended. Different management factors and treatment and segregation strategies can then be the cause of variations in bulk tank milk antibody levels in herds experiencing an outbreak of $M$. bovis-associated disease. For this reason, bulk tank milk sampling is less suitable for identification of $M$. bovis-infected herds than for other pathogens, which elicit stronger antibody responses in subclinically infected cattle.

\section{Cows with Nonspecific or No M. bovis- Associated Disease}

To be as certain as possible that the cows in the SYS group represented $M$. bovis-infected animals, and that cows in the ND group were not infected with $M$. bovis, an intermediate group-NS - was required. Cows in this group may include cows that are infected with $M$. bovis without showing clear clinical signs, cows that were infected earlier and are no longer showing clinical signs, and cows that are not infected. It is therefore difficult to make firm conclusions regarding dynamics in this group, and we note that the ODC\% was generally estimated by the model as being somewhere between that of the ND and SYS groups (although closer to the former).

Herd 1 was overrepresented in the NS group. This could be because herd visits were initiated for herd 1 later in the outbreak than in the other herds, so some cows might have shown clinical signs earlier and were recovering by the time of the herd visit. This could explain the intermediate antibody responses in many cows in this disease group and the slightly declining mean predicted ODC\% (Figure 3).

Two cows from the NS and ND groups stood out as having high ODC\% in milk (Figures $2 \mathrm{C}$ and $2 \mathrm{D}$ ). The most likely explanation for these must be undiagnosed or subclinical mastitis (Fox, 2012), because records in 
the electronic herd recording system did not reveal former treatment of mastitis. Given the fact that the mean for the NS group was closer to the mean of the ND group than to that of the SYS group, it is more likely that these cows were either not infected or were previously infected with $M$. bovis.

\section{Study Design and Limitations}

The aim of this study was to investigate the dynamics of antibodies directed against $M$. bovis under field conditions. Advice about how to handle the disease outbreak or specific cows was not given by any of the project veterinarians, and decisions regarding treatment and herd-specific disease control strategies were made solely by the farmer and his or her consulting veterinarian. We cannot be sure that our presence and interest in sampling did not influence decisions about strategy, but it is not our impression that this happened to a great extent. However, culling strategies varied between herds due to different local veterinary advice and differences in clinical signs and severity of disease.

In addition, we note that the predefined test-positive cut-off value of the BioX ELISA (37 ODC\%) has not yet been substantially documented in naturally infected cattle. To evaluate which cut-off is meaningful, an overview of the variation between individual cows, as provided here, is needed as the first step. However, the proportion $(\mathrm{P})$ of test results that would be expected to exceed a given arbitrary threshold based on our results can be calculated as follows:

$$
\mathrm{P}\left(+\mid \mu_{g, d}, \sigma_{a}, \sigma_{r}, t\right)=1-\Phi\left(\frac{\log (t+1)-\log \left(\mu_{g, d}\right)}{\sqrt{\sigma_{a}^{2}+\sigma_{r}^{2}}}\right),
$$

where $\mu_{g, d}$ is the estimated mean value for group $g$ at time $d$ (Figures 3 and 4 ), $\sigma_{a}$ and $\sigma_{r}$ are the standard deviations associated with cow/herd and residual for the same group $g$ (Table 4), $t$ is the desired test threshold (e.g., 37 ODC\%), and $\Phi$ is the standard normal distribution.

\section{CONCLUSIONS}

To our knowledge, this is the first longitudinal observational field study of naturally infected herds in which the dynamics of the IgG response directed against $M$. bovis in dairy cows are described and compared between groups of animals with different disease manifestations. We conclude that antibody measurements in milk are only useful for differential diagnosis of mastitis and that serum antibody responses are highly dynamic and variable between individual cows. Consequently, serology based on the BioX Bio K 302 ELISA is not likely to be useful for diagnosis of $M$. bovis-associated disease in individual dairy cows. However, this does not preclude use of the test for herd- or group-level diagnosis, which is supported by the fact that the estimated mean of the measured antibody response was markedly higher in the group of cows with $M$. bovis-associated systemic disease than in those without $M$. bovis-associated disease between 7 and $17 \mathrm{~d}$ after disease onset in particular. Further studies are warranted to identify optimal herd-level testing strategies based on the information provided here.

\section{ACKNOWLEDGMENTS}

The authors thank veterinarians Ulla Torpe and Martin Lund (Dyrlægerne Egtved), Lars Pedersen (SEGES, Aarhus, Denmark), and veterinarians/veterinary students Annie Nielsen (Dyrlægerne Optivet), Eva Elisabeth Toft-Petersen (Tinglev Dyrehospital), Franziska Helene Pedersen (University of Copenhagen), and Signe Rejnhardt Olsen (Skovbjerg Dyrlæge Team) for assistance at the herd visits. The Danish Milk and Cattle Levy Funds provided funding for the sampling and laboratory testing. The funding source was not involved in the study design, analyses, and interpretation, nor in the decision to publish the study.

\section{REFERENCES}

BioX Diagnostics. 2017. Mycoplasma bovis ELISA kit. Nov. 17, 2017. http://www.biox.com/en/bio-k-302-monoscreen-abelisa -mycoplasma-bovis-indirect-monowell-p-250/.

Boothby, J. T., D. E. Jasper, and C. B. Thomas. 1986. Experimental intramammary inoculation with Mycoplasma bovis in vaccinated and unvaccinated cows: Effect on the mycoplasmal infection and cellular inflammatory response. Cornell Vet. 76:188-197.

Boothby, J. T., D. E. Jasper, and C. B. Thomas. 1987. Experimental intramammary inoculation with Mycoplasma bovis in vaccinated and unvaccinated cows: Effect on local and systemic antibody response. Can. J. Vet. Res. 51:121-125.

Bürki, S., J. Frey, and P. Pilo. 2015. Virulence, persistence and dissemination of Mycoplasma bovis. Vet. Microbiol. 179:15-22.

Byrne, W., B. Markey, R. McCormack, J. Egan, H. Ball, and K. Sachse. 2005. Persistence of Mycoplasma bovis infection in the mammary glands of lactating cows inoculated experimentally. Vet. Rec. 156:767-771.

Byrne, W. J., H. J. Ball, N. Brice, R. McCormack, S. E. Baker, R. D. Ayling, and R. A. Nicholas. 2000. Application of an indirect ELISA to milk samples to identify cows with Mycoplasma bovis mastitis. Vet. Rec. 146:368-369.

Fox, L. K. 2012. Mycoplasma mastitis: Causes, transmission, and control. Vet. Clin. North Am. Food Anim. Pract. 28:225-237. https:// doi.org/10.1016/j.cvfa.2012.03.007.

Fredriksen, B., T. Sandvik, T. Loken, and S. A. Odegaard. 1999. Level and duration of serum antibodies in cattle infected experimentally and naturally with bovine virus diarrhoea virus. Vet. Rec. 144:111-114.

Hazelton, M. S., P. A. Sheehy, K. L. Bosward, A. M. Parker, J. M. Morton, C. J. Dwyer, P. G. Niven, and J. K. House. 2018. Short 
communication: Shedding of Mycoplasma bovis and antibody responses in cows recently diagnosed with clinical infection. J. Dairy Sci. 101:584-589.

Henderson, J. P., and H. J. Ball. 1999. Polyarthritis due to Mycoplasma bovis infection in adult dairy cattle in Northern Ireland. Vet. Rec. 145:374-376.

Howard, C. J., K. R. Parsons, and L. H. Thomas. 1986. Systemic and local immune responses of gnotobiotic calves to respiratory infection with Mycoplasma bovis. Vet. Immunol. Immunopathol. 11:291-300.

Le Grand, D., D. Calavas, M. Brank, C. Citti, R. Rosengarten, P. Bezille, and F. Poumarat. 2002. Serological prevalence of Mycoplasma bovis infection in suckling beef cattle in France. Vet. Rec. 150:268-273

Martin, S. W., K. G. Bateman, P. E. Shewen, S. Rosendal, J. G. Bohac, and M. Thorburn. 1990. A group level analysis of the associations between antibodies to seven putative pathogens and respiratory disease and weight gain in Ontario feedlot calves. Can. J. Vet. Res. 54:337-342.

Maunsell, F. P., and G. A. Donovan. 2009. Mycoplasma bovis infections in young calves. Vet. Clin. North Am. Food Anim. Pract. 25:139-177.

Maunsell, F. P., A. R. Woolums, D. Francoz, R. F. Rosenbusch, D. L. Step, D. J. Wilson, and E. D. Janzen. 2011. Mycoplasma bovis infections in cattle. J. Vet. Intern. Med. 25:772-783.

Nicholas, R. A. 2011. Bovine mycoplasmosis: Silent and deadly. Vet. Rec. 168:459-462.

Nicholas, R. A., R. D. Ayling, and L. P. Stipkovits. 2002. An experimental vaccine for calf pneumonia caused by Mycoplasma bovis: clinical, cultural, serological and pathological findings. Vaccine 20:3569-3575.

Nielsen, L. R. 2003. Salmonella Dublin in dairy cattle: Use of diagnostic tests for investigation of risk factors and infection dynamics. $\mathrm{PhD}$ Thesis. The Royal Veterinary and Agricultural University, Frederiksberg, Denmark.

Nielsen, P. K., M. B. Petersen, L. R. Nielsen, T. Halasa, and N. Toft. 2015. Latent class analysis of bulk tank milk PCR and ELISA testing for herd level diagnosis of Mycoplasma bovis. Prev. Vet. Med. 121:338-342.
Parker, A. M., J. K. House, M. S. Hazelton, K. L. Bosward, J. M. Morton, and P. A. Sheehy. 2017. Bulk tank milk antibody enzymelinked immunosorbent assay as a biosecurity tool for detecting dairy herds with past exposure to Mycoplasma bovis. J. Dairy Sci. 100:8296-8309.

Petersen, M. B., K. Krogh, and L. R. Nielsen. 2016. Factors associated with variation in bulk tank milk Mycoplasma bovis antibodyELISA results in dairy herds. J. Dairy Sci. 99:3815-3823.

R Core Team. 2016. R: A language and environment for statistical computing. R Foundation for Statistical Computing, Vienna, Austria. https://www.R-project.org/.

Rosendal, S., and S. W. Martin. 1986. The association between serological evidence of mycoplasma infection and respiratory disease in feedlot calves. Can. J. Vet. Res. 50:179-183.

Szacawa, E., K. Niemczuk, K. Dudek, D. Bednarek, R. Rosales, and R. Ayling. 2015. Mycoplasma bovis infections and co-infections with other Mycoplasma spp. with different clinical manifestations in affected cattle herds in eastern region of Poland. Bull. Vet. Inst. Pulawy 59:331-337.

Szacawa, E., M. Szymanska-Czerwinska, K. Niemczuk, K. Dudek, D. Bednarek, and R. D. Ayling. 2016. Comparison of serological, molecular and cultural diagnostic methods for the detection of $\mathrm{Myco-}$ plasma bovis infections in cattle. Anim. Sci. Pap. Rep. 60:391-397.

Wawegama, N. K., and G. F. Browning. 2017. Improvements in diagnosis of disease caused by Mycoplasma bovis in cattle. Anim. Prod. Sci. 57:1482-1487.

Wawegama, N. K., P. F. Markham, A. Kanci, M. Schibrowski, S. Oswin, T. S. Barnes, S. M. Firestone, T. J. Mahony, and G. F. Browning. 2016. Evaluation of an IgG enzyme-linked immunosorbent assay as a serological assay for detection of Mycoplasma bovis infection in feedlot cattle. J. Clin. Microbiol. 54:1269-1275.

Wilson, D. J., R. T. Skirpstunas, J. D. Trujillo, K. B. Cavender, C. V. Bagley, and R. L. Harding. 2007. Unusual history and initial clinical signs of Mycoplasma bovis mastitis and arthritis in firstlactation cows in a closed commercial dairy herd. J. Am. Vet. Med. Assoc. 230:1519-1523.

Wood, S. N. 2011. Fast stable restricted maximum likelihood and marginal likelihood estimation of semiparametric generalized linear models. J. R. Stat. Soc. B 73:3-36. 


\section{APPENDIX}

Table A1. Standardized clinical protocol for examination of selected cows focusing on the respiratory system, udder, and musculoskeletal system

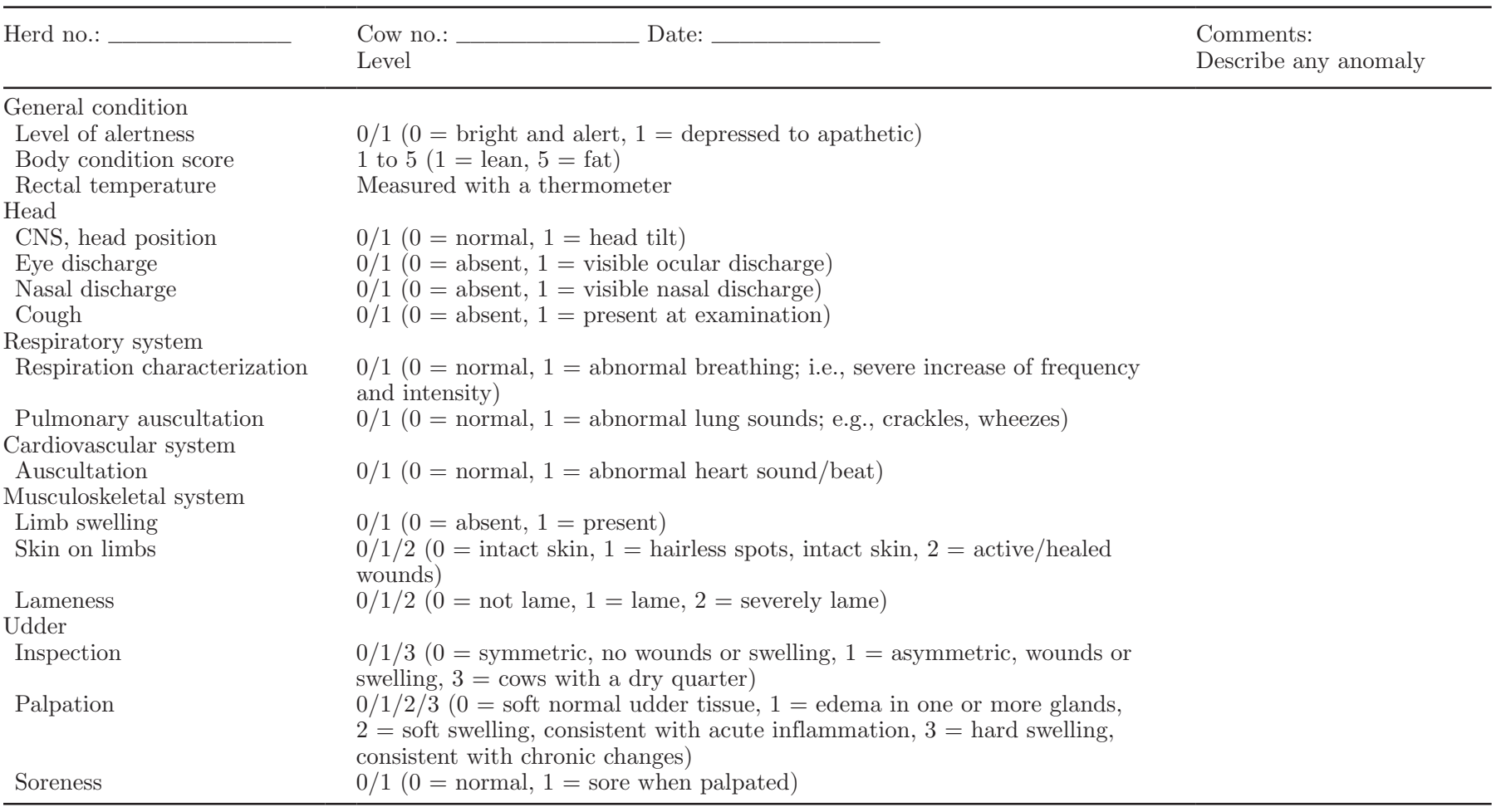

